\title{
Topology and Causal Structure
}

\author{
Andrew Chamblin \\ Department of Applied Mathematics and Theoretical Physics, \\ University of Cambridge, Cambridge CB3 9EW, England
}

\begin{abstract}
We investigate the causal structure of spacetimes $(M, g)$ for which the metric $g$ is singular on a set of points. In particular, we show that it is always possible to continuously deform the metric so that the resulting spacetime is causal.

Furthermore, we develop the framework of $(2+1)$-dimensional pure gravity to include processes describing the creation and annihilation of gravitational skyrmions, or 'kinks'. In this approach, a sector in the 'Fock space' is classified by purely topological data: The topology of the triad and of the manifold in the asymptotic region. We use this construction to generalise the selection rule of Amano and Higuchi [5] to a selection rule governing when the amplitude for a given process vanishes. We present various applications and examples of such processes.
\end{abstract}

\section{Metric Topology}

Statements about the kinematical aspects of causal structure have long been of interest to those working in classical and quantum gravity ([1], [2], [3], [4]). For the most part, these studies have focussed on the causal structure of spacetimes $(M, g)$ for which the metric $g$ is globally non-singular. However, the work of a number of authors ([7], [8], [9], [10]) suggests that degenerate metrics may be a generic feature of any quantum theory of gravity which incorporates topology changing processes. With this in mind, it is natural to investigate the causal properties of spacetimes with degenerate Lorentzian structures. In order to fully appreciate this issue, we need to first define, in a detailed way, what a 'singularity' in the metric is, and how the 'index' of a singularity is associated with the topology of the metric. 
To begin with, let us assume that $(M, g)$ is $n$-dimensional and timeorientable [11]. It follows [5] that we can express the Lorentz metric, $g_{a b}^{L}$, in terms of some auxilliary Riemannian metric $g_{a b}^{R}$ and some timelike vector field $V$, in the usual way:

$$
g_{a b}^{L}=g_{a b}^{R}-2 V_{a} V_{b}
$$

where in equation (1), $g_{a b}^{R}$ is some (arbitrary) Riemannian metric on $M$, and $V$ has been normalised with respect to $g_{a b}^{R}$, that is, $g_{a b}^{R} V^{a} V^{b}=1$. Thus, there is a correspondence between vector fields and Lorentz metrics on $M$.

Now, a natural question is: Given two Lorentz metrics, $g$ and $g^{\prime}$ (on $M$ ), when can $g^{\prime}$ be obtained from $g$ by a continuous deformation? This question should be relevant in any Lorentzian sum-over-histories version of quantum gravity, since if two metrics $g$ and $g^{\prime}$ are related by some element $\phi$ of the identity connected component of the diffeomorphism group it follows that the two metrics must also be homotopic. In the light of the above correspondence between timelike vector fields and Lorentz metrics, we see that this question reduces to the question: Given two vector fields, $V$ and $V^{\prime}$ (on $M$ ), when can $V^{\prime}$ be obtained from $V$ by continuous deformation? This question is well understood [6]; it turns out that the answer depends upon the degrees of certain maps (defined by the vector fields $V$ and $V^{\prime}$ ) from certain $(n-1)$-dimensional submanifolds of $M$, into $S^{n-1}$. In the context of General Relativity, the degree of such a map is often called the 'kink number' [12]. We need to describe the kink number in more detail.

First, we let $S(M)$ denote the unit-sphere bundle over $M$. That is, at each $p \in M$, the fibre, $S_{p}(M)$, of $S(M) \longrightarrow M$ is the ' $n$ - 1 -sphere of directions' at $p$. Since $\operatorname{dim}\left(S_{p}(M)\right)=n-1$ and $\operatorname{dim}(M)=n$, we see that the dimension of the bundle space $S(M)$ is $2 n-1$. Let $\Sigma$ denote any connected, closed $(n-1)$-dimensional submanifold of $M$. Then we can restrict the bundle $S(M) \longrightarrow M$ to $\Sigma$, obtaining the $2 n-2$ dimensional bundle $S(\Sigma) \longrightarrow \Sigma$, the $S^{n-1}$-bundle over $\Sigma$. Since $(M, g)$ is time-orientable, we can naturally choose a unit normal vector field, $n$, to $\Sigma$. Clearly, $n$ determines a section of $S(\Sigma)$ : $n \in \Gamma(M, S(\Sigma))$. Likewise, let $V$ be any future timelike vector associated with the Lorentz metric $g_{a b}^{L}$ (as in equation (1)), then the restriction of $V$ to $\Sigma$ also yields a section $V \in \Gamma(M, S(\Sigma))$. These two sections, $n$ and $V$, will generically intersect at a finite number of points $\chi_{i} \in S(\Sigma), i=1, \ldots m$. We 
assign each intersection point a sign, $\operatorname{sign}\left(\chi_{i}\right)$, defined by

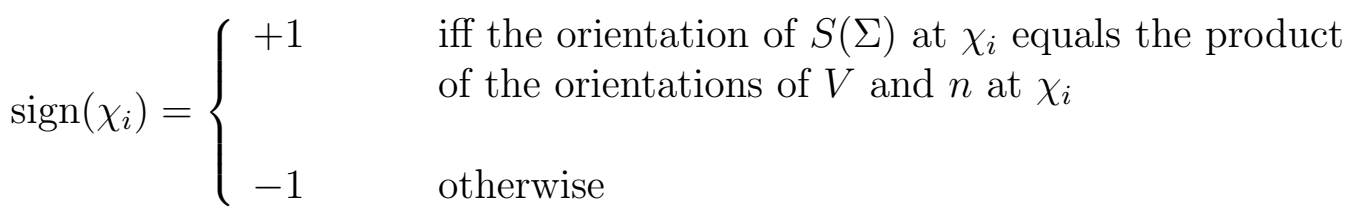

We can then define the kink number of the timelike vector field $V$ with respect to the three-surface $\Sigma$ (denoted ' $\operatorname{kink}(\Sigma ; V)$ ') by the formula

$$
\operatorname{kink}(\Sigma ; V)=\sum_{i=1}^{m} \operatorname{sign}\left(\chi_{i}\right)
$$

As discussed above, the topology of the Lorentz metric $g_{L}$ is equivalent to the topology of the timelike vector field $V$, which in terms of the kink number simply means that

$$
\operatorname{kink}(\Sigma ; V)=\operatorname{kink}\left(\Sigma ; g_{L}\right)
$$

With this definition of metric homotopy, we can now discuss the topology of metric singularities.

In this paper, we shall only consider the situation where the metric $g_{L}$ fails to be invertible on a collection of points, $p_{i} \in M$; we shall refer to such a point where the metric is not invertible as a singularity. We should point out that this sort of singularity is highly non-generic, since in general the metric will fail to be invertible on higher dimensional hypersurfaces in the spacetime. We adopt this definition of a singularity purely as a simplifying assumption. Using equation (1), we see that a metric singularity is thus present at any point $p \in M$ at which the timelike vector field, $V$, becomes singular, i.e., $V$ must vanish identically at $p$. The topological nature of vector field singularities is well-understood [6]. To each singularity, $p$, of a vector field $V$ on $M$, one assigns an index, defined as follows:

Let $S_{\varepsilon}^{n-1}(p)$ be a tiny $(n-1)$-dimensional sphere (of radius $\varepsilon$ ) at $p$. Since the vector field $V$ is assumed to vanish only at $p$ (and not on a neighbourhood of $p), V$ is non-vanishing on $S_{\varepsilon}^{n-1}(p)$. $V$ thus defines a map from $S_{\varepsilon}^{n-1}(p)$ to $S^{n-1}$, and we define the index of $V$ at $p$ (denoted $\left.\operatorname{ind}(V, p)\right)$ by the formula

$$
\operatorname{ind}(V, p)=1-\operatorname{kink}\left(S_{\varepsilon}^{n-1}(p) ; V\right)
$$


In analogy with equation (3), we therefore define the index of a Lorentz metric, $g_{L}$, at a metric singularity $p$ by the relation

$$
\operatorname{ind}\left(g_{L}, p\right)=1-\operatorname{kink}\left(S_{\varepsilon}^{n-1}(p) ; g_{L}\right)
$$

We now illustrate the rough characteristics of different index singularities as an aid to the visual imagination.

Index 1: If the metric $g_{L}$ has index equal to 1 at some point $p \in M$, it follows from equation (5) that $\operatorname{kink}\left(S_{\varepsilon}^{n-1}(p) ; g_{L}\right)=0$, for any little $(n-1)$ sphere about $p$. Intuitively, this means that the light cones do not 'spin around' as one approaches the singularity $p$. Below, we have drawn three typical exampes of index 1 singularities, which we call 'bang', 'crunch', and 'flush' singularities for obvious reasons: Fig. 1. 
Three simple examples of index 1 singularities.

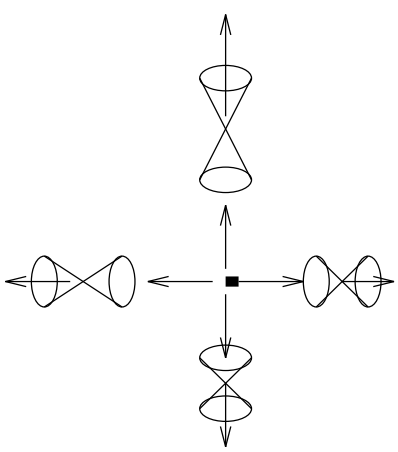

A 'bang'.

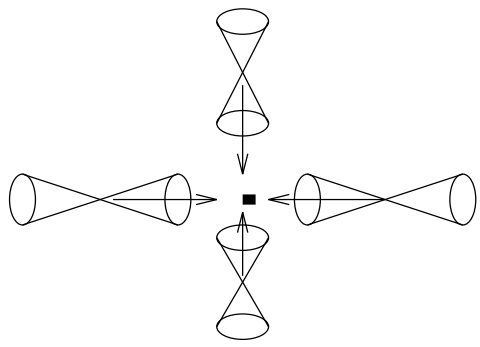

A 'crunch'.

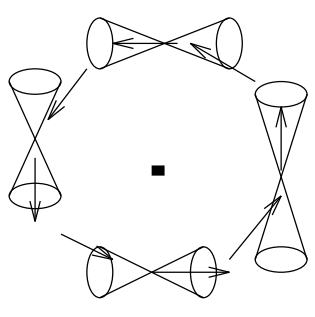

A 'flush'.

Figure 1

Index 2: When the index of a metric singularity $p$ is 2 , the light cone field must kink once 'negatively' on $S_{\varepsilon}^{n-1}(p)$, i.e.,

$$
\operatorname{kink}\left(S_{\varepsilon}^{n-1}(p) ; g_{L}\right)=-1
$$

A simple example of an index 2 singularity is the 'dipole' singularity. In the below figure, we show how an index 2 singularity can be obtained from two index 1 singularities by continuously deforming the two index 1 singularities until they 'hit' each other: Fig. 2. 


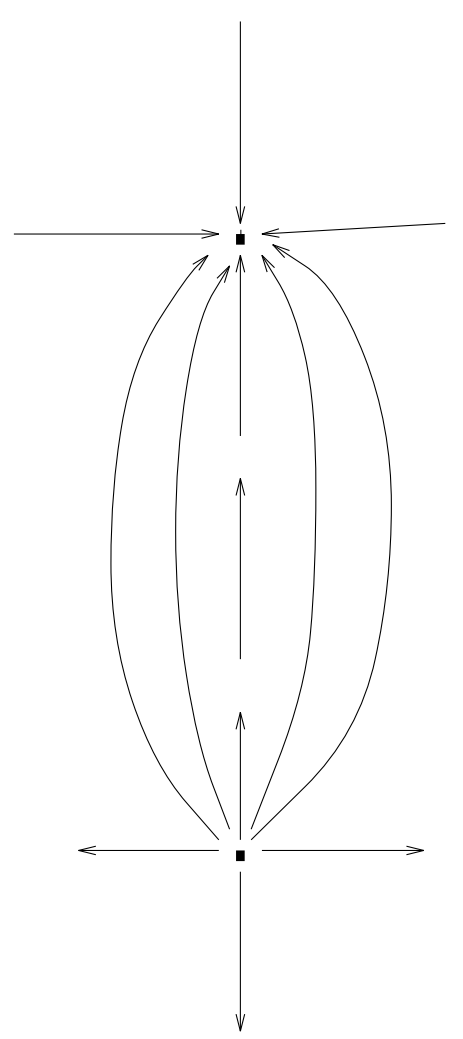

Begin with two index 1 singularities.

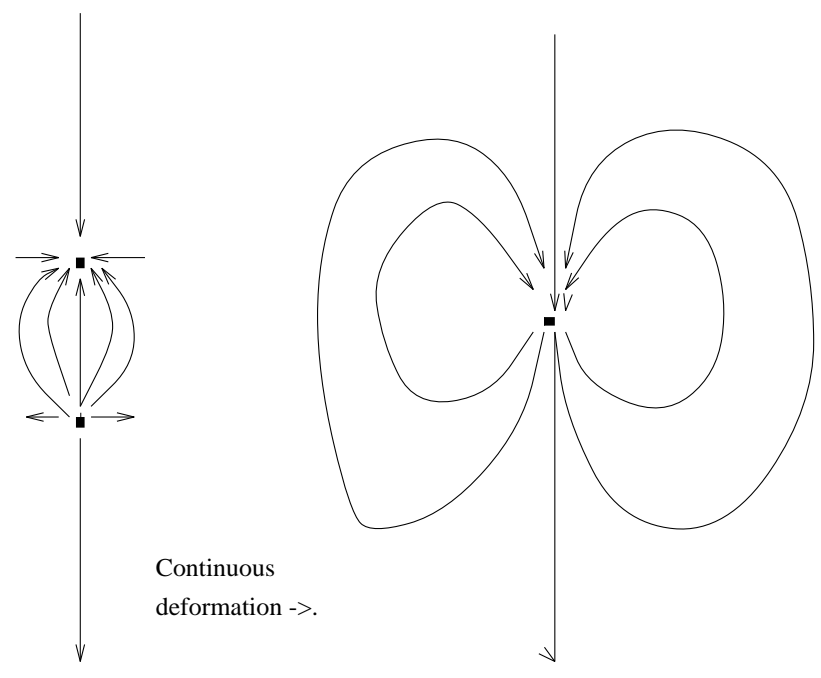

Crash them together.
Get an index 2 singularity.

Figure 2

Index -1: A metric $g_{L}$ with an index -1 singularity $p$, must have $\operatorname{kink}\left(S_{\varepsilon}^{n-1}(\right.$ $\left.p) ; g_{L}\right)=2$. Interestingly, this is precisely the type of singularity which occurs in the Deutsch-Politzer time machine in even spacetime dimensions [14]. We therefore refer the reader to [14] for an example of this type of singularity.

Higher and higher index singularities can be readily constructed by generalising the constructions illustrated above (for example, by 'throwing together' a bunch of index 1 singularities). This can be an amusing exercise, for some people. The point is, there is no difference (up to a continuous deformation) between an index 1 singularity, and a pair of singularities, one with 
index 597 and the other with index -596 (since we can 'crash' them together to get the index 1 singularity back). On the other hand, we do not really feel that index 597 singularities are 'generic'. In fact, index 1 singularities seem almost 'natural', and at least have the distinction of appearing in the cosmology literature where one talks of big 'bangs' and 'crunches' (although we have yet to hear of a 'big flush').

On the other hand, there is a real sense in which the 'crashing together' of point singularities is not a physical process. After all, a singularity should presumably be thought of as a component of the boundary of the spacetime; pushing two singularities together would therefore seem to alter the nature of the boundary. Since the boundary of a compact spacetime contributing to a functional integral is usually assumed to comprise the 'initial' and 'final' states (before and after 'scattering') then spacetimes with different boundaries must correspond to completely different processes. We should therefore outlaw deformations which move singularities together. Indeed, this leads to an interesting way of thinking about these point singularities: Since a neighborhood of any singularity is a portion of spacetime homeomorphic to $S^{n-1} \times \mathbb{R}$, it makes sense to think of a singularity as an 'asymptotic state' corresponding to an 'incoming' (or 'outgoing') $S^{n-1}$ with a kink on it (we will have more to say about kinky boundary conditions later). With this in mind, whenever we consider a 'continuous deformation of a metric' we shall always mean a deformation which does not move any metric singularities together.

\section{Causality and Singularities}

To begin with, we recall the precise statement of Geroch's result:

Theorem. (Geroch [1]) Let $\left(M, g_{L}\right)$ be a compact, time-orientable spacetime with globally non-singular Lorentz metric $g_{L}$. Suppose that the boundary of $M$ is equal to the disjoint union of two compact spacelike three-manifolds, $\partial M \cong \Sigma_{1} \cup \Sigma_{2}$, and that there are no closed timelike curves in $\left(M, g_{L}\right)$. Then $\Sigma_{1}$ and $\Sigma_{2}$ are diffeomorphic, and $M$ is topologically of the form $\Sigma_{1} \times[0,1]$.

Thus, if $\Sigma_{1}$ and $\Sigma_{2}$ are not even homeomorphic, there must exist CTCs in $\left(M, g_{L}\right)$ (we shall assume time-orientability throughtout this paper). Of 
course, if we allow the metric $g_{L}$ to be singular, then the above result will not in general hold. As Geroch noted in his original proof of the above Theorem, the timelike flow (induced by $g_{L}$ ) will induce a diffeomorphism from $\Sigma_{1}$ to $\Sigma_{2}$ unless the timelike curves close back on themselves or they hit singularities.

A natural question, then, is whether or not it is always possible to arrange for all the CTCs to hit singularities. Given a compact causality violating spacetime $\left(M, g_{L}\right)$ (with or without boundary), such that $g_{L}$ is singular at some finite collection of points $\left\{p_{1}, p_{2}, \ldots p_{n}\right\}$, can we always 'deform' all of the CTCs so that they 'hit' the singularities? The answer is yes. In fact, by slightly modifying the arguments of [3], we can prove

Theorem 1. Let $M$ be a smooth compact n-manifold, without boundary, and let $g_{L}$ be some Lorentz metric on $M$ such that $g_{L}$ is singular on a finite collection of points $\left\{p_{1}, p_{2}, \ldots p_{m}\right\}$ in $M$ and $\left(M, g_{L}\right)$ is causality violating. Then there always exists a new metric, $g_{L}^{\prime}$, which can be obtained by a continous deformation of the original metric $g_{L}$ such that $\left(M, g_{L}^{\prime}\right)$ is causal.

Proof. Given a point, $p_{i} \in M$, at which $g_{L}$ is singular, let $B_{\varepsilon}^{n}\left(p_{i}\right)$ denote the closed $n$-ball of radius $\varepsilon$ about $p_{i}$ and $S_{\varepsilon}^{n-1}\left(p_{i}\right) \cong \partial B_{\varepsilon}^{n}\left(p_{i}\right)$, as above. Let $N$ be obtained from $M$ by removing all of the $B_{\varepsilon}^{n}\left(p_{i}\right)$ :

$$
N \cong M-\left(B_{\varepsilon}^{n}\left(p_{1}\right) \cup B_{\varepsilon}^{n}\left(p_{2}\right) \ldots \cup B_{\varepsilon}^{n}\left(p_{m}\right)\right)
$$

Then, by construction, the spacetime $\left(N, g_{L}\right)$ is non-singular and open (since the boundary, $\partial N$, does not lie in $N$; this is the main technical difference from the construction in [3]). We wish to 'cut' all of the CTCs in $\left(N, g_{L}\right)$ by deforming them onto $\partial N$, as outlined in [3]. Since $\partial N$ is not in $N$, this is a bit tricky. Technically, we need to briefly pass to $\left(\bar{N}, g_{L}\right)$, where $\bar{N}$ denotes the closure of $N .\left(\bar{N}, g_{L}\right)$ is then a compact spacetime with boundary $\partial N$. As in [3], we can cover $\bar{N}$ with a finite number of sets of the form

$$
S_{q_{i}}=\left\{x \in I^{+}\left(q_{i}\right) \cap I^{-}(q) \mid q \in I^{+}\left(q_{i}\right)\right\}
$$

and we can take the sets in this cover to be locally causal (no CTC lies entirely in any one of the $S_{q_{i}}$ ). As in [3], we can use these sets to construct a continuous deformation of the metric $g_{L}$ on $\bar{N}$ to a new metric $g_{L}^{\prime}$, such that all of the CTCs are pushed onto the boundary, $\partial N$. We now pass back to $\left(N, g_{L}^{\prime}\right)$. We have thus pulled all of the CTCs arbitrarily close to the singular 
points, as shown: Fig. 3.

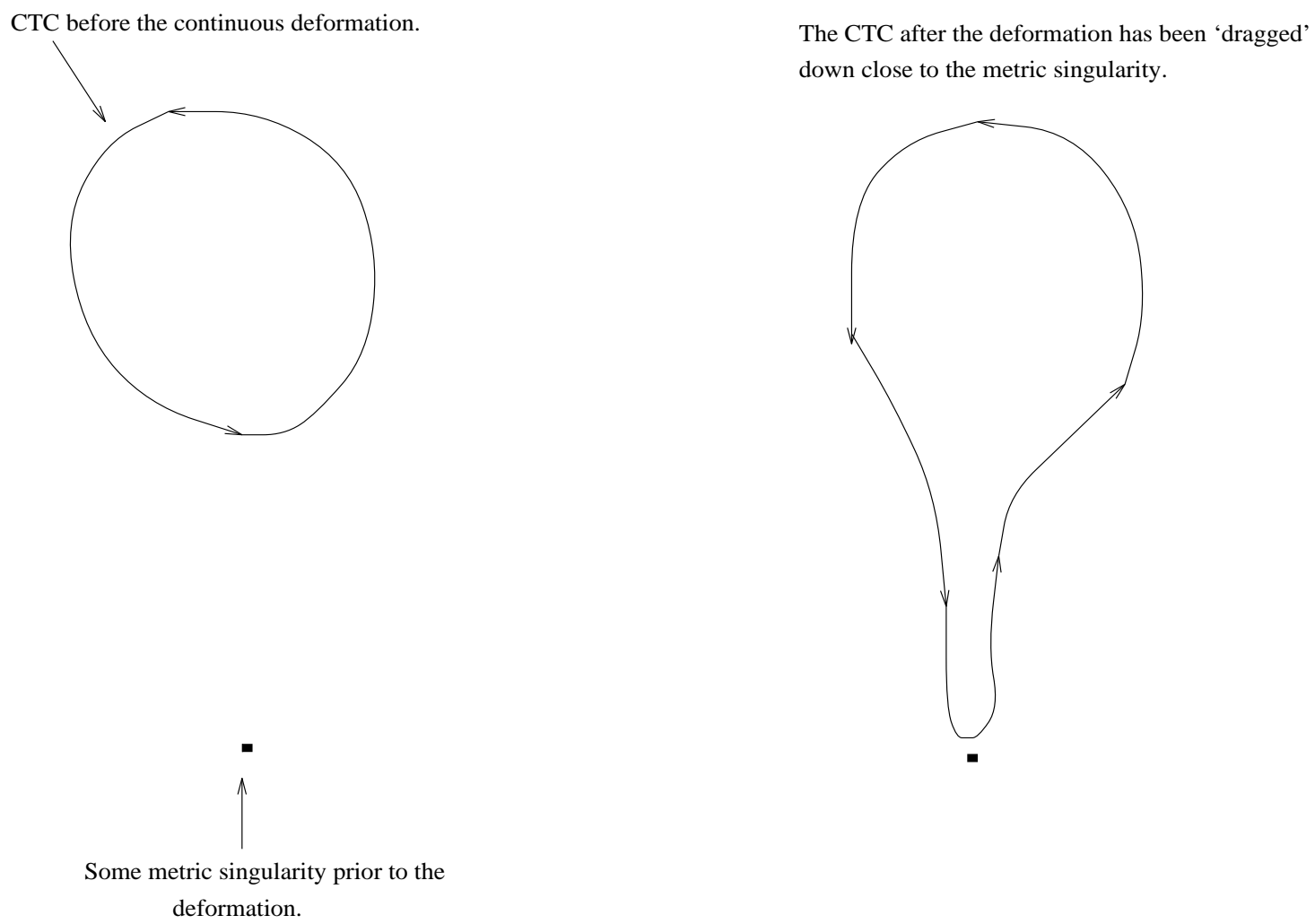

Figure 3

Furthermore, since the deformation is continuous, the kink number on each $S_{\varepsilon}^{n-1}\left(p_{i}\right)$ is unchanged, that is, we have not altered the index of any singularity. We then continue this construction in the limit $\varepsilon \longrightarrow 0$, so that all CTCs are continuously forced onto the singular points. We have therefore constructed a continuous deformation, $g_{L} \longrightarrow g_{L}^{\prime}$, such that $\left(M, g_{L}^{\prime}\right)$ is causal.

Thus, given a singular spacetime with CTCs, we can always 'destroy' the CTCs using the singularities. Furthermore, by construction the deformation 
does not move any of the singularities together.

Of course, one could take the view that spacelike topology change is too restrictive and that the topology of the metric should also be allowed to fluctuate. In the context of a 'Lorentzian' quantum gravity theory obtained by a path integral prescription, this would mean that instead of calculating the probability amplitude to pass from some initial spacelike three-manifold to some final spacelike three-manifold, one would instead seek an 'amplitude' describing the probability of transition 'from' a three-manifold $\Sigma_{1}$, such that the Lorentz metric $g_{L}$ has kink number $n_{1}$ on $\Sigma_{1}$, 'to' a three-manifold $\Sigma_{2}$, such that the Lorentz metric $g_{L}$ has kink number $n_{2}$ on $\Sigma_{2}$. In this way, the 'boundary data' (or asymptotic states) of such a theory would be purely topological. We stress this viewpoint (even though it goes against the spirit of canonical quantum gravity) since we feel it is nonsensical to study strictly spacelike topology change via spacetimes which have metric singularities. To see why, let $\left(M, g_{L}\right)$ be any spacetime with spacelike boundary $\partial M$ and let $g_{L}$ be singular at $p$. We then obtain a spacetime $\left(M, g_{L}^{\prime}\right)$, with a kink in $g_{L}^{\prime}$ on $\partial M$, simply be deforming the singular point $p$ across $\partial M$, as shown: Fig. 4 . 


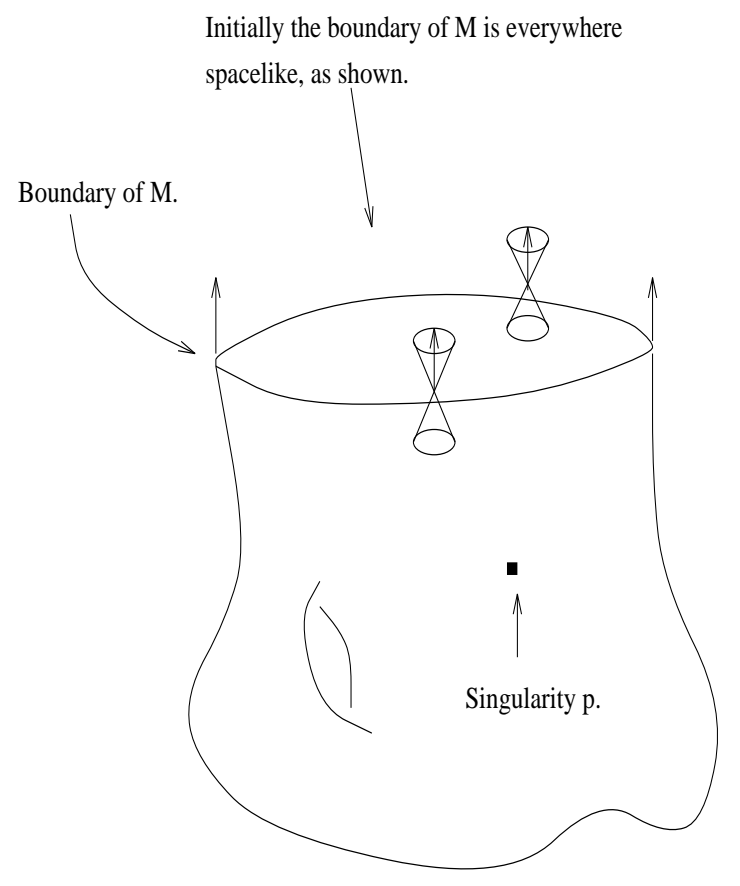

Initially we assume that $(\mathrm{M}, \mathrm{g})$ has spacelike boundary and that there is a singularity in the metric at the point $\mathrm{p}$.

Figure 4
After the deformation the metric has nontrivial homotopy with respect to the boundary of $\mathrm{M}$.

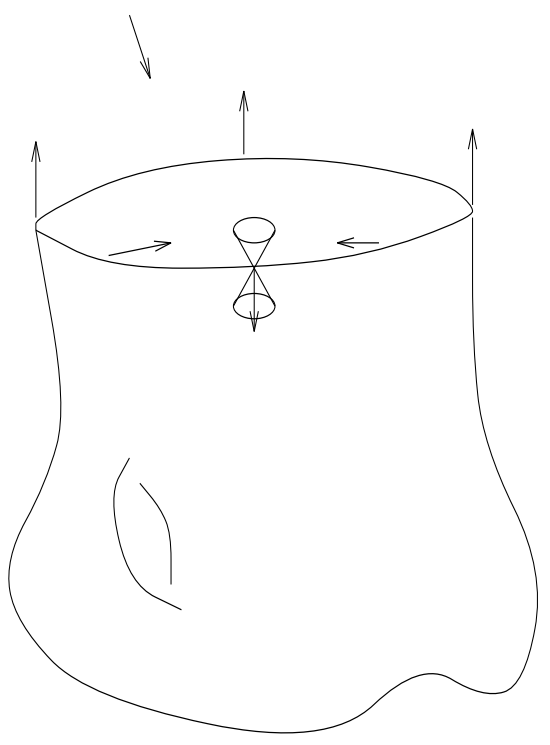

After we continuously move the singularity across the boundary of $\mathrm{M}$ we find that there must now be a kink in the light cone field on the boundary of M. Thus, the annihilation of the singularity has resulted in the creation of a kink, or gravitational skyrmion.

The point is, the continuous deformation could perhaps represent the 'flow of time'. The creation/annihilation of singularities corresponds to creation/annihilation of kinks (note that in the above example, one would have $\left.\operatorname{kink}\left(\partial M ; g_{L}^{\prime}\right)=\operatorname{ind}\left(g_{L}, p\right)\right)$. The two concepts are dual in this sense (see also [18] for ideas along these lines). This point of view also fits in nicely with our previous idea that a singularity can (to a certain extent) be regarded as an 'internal' asymptotic state. We now present an example of a model where the production and annihilation of kinks can be explicitly realised: $(2+1)$ dimensional gravity. 


\section{The Model in $(2+1)$ Dimensions}

Attempts to quantise gravity via a path integral or sum over histories formalism have led to many fascinating insights and results. One of the most interesting and well-known constructions to emerge from these efforts is Witten's $(2+1)$-dimensional toy model ([10], [19], [20]). In this approach, one first writes down the theory in the language of fibre bundles. That is, one identifies, as the fundamental variables, a triad $e^{a}$ and a spin $(S O(2,1))$ connection $w^{a}$. The triad, which explicitly is just a collection of three linearly independent 1-forms, $e_{\mu}^{0}, e_{\mu}^{1}$ and $e_{\mu}^{2}$, will in general be degenerate. If we recover the Lorentz metric by the usual formula

$$
g_{\mu \nu}=\eta_{a b} e_{\mu}^{a} e_{\nu}^{b} \quad\left(\eta_{a b}=\operatorname{diag}(-1,+1,+1)\right),
$$

we see that the subsets of $M$ at which $e_{\mu}^{0}$ (the 'timelike' vector in the triad) vanishes will be sets at which the metric $g_{\mu \nu}$ fails to be invertible. A partial analysis of the causal structure of such degenerate spacetimes was given above (although only for the case where the singular set is a collection of points). The point is, only by allowing such singular behaviour can we complete the identification of pure gravity as a gauge theory in $(2+1)$ dimensions [19].

Having decided on a kinematical framework, the next step is to write down the Einstein-Hilbert action

$$
\mathcal{S}_{E H}[M]=\int_{M} e^{a} \wedge\left(d w_{a}+\frac{1}{2} \varepsilon_{a b c} w^{b} \wedge w^{c}\right)=\int_{M} e^{a} \wedge R_{a}
$$

Thus, the partition function, $Z(M)$, associated with any closed three-manifold $M$ (with or without boundary) is given abstractly by the path integral

$$
Z(M)=\int \mathcal{D} w \mathcal{D} e e^{i \int_{M} e^{a} R_{a}}
$$

Integrating over the space of triad fields $e$, one obtains [19]

$$
Z(M)=\int \mathcal{D} w \delta(R)
$$

where, as in equation $(7), R$ is the $\mathfrak{s o}(2,1)$-valued curvature 2 -form. Of cru-

cial importance are the boundary conditions one imposes on the connection 
1-forms which appear in the sum (8). That is, for a closed three-manifold $M$ with boundary $\partial M$, the data on $\partial M$ corresponds to 'asymptotic states'. The most common approach is to impose the condition that the sum in equation (8) is only over flat connections which induce spacelike data on $\partial M$ (recall that the sum in (8) is over flat connections, since the field equations induced by equation (6) imply that the $\delta$-function in equation (8) has support only on those connections for which $R(w)=0)$. The condition that $\partial M$ is spacelike relative to the data $(e, w)$ is tantamount to the assumption that $w$ induces an $S O(2)$ (or $U(1)$ ) valued connection 1-form $\left.w\right|_{\partial M}$ on $\partial M$. Amano and Higuchi [21] have shown that imposing these boundary conditions in the sum (8) leads to a selection rule which governs when the amplitude for a given topology-changing process is non-vanishing, i.e. $Z(M) \neq 0$. One of the principal aims of this paper is to allow for $w$ to have arbitrary boundary conditions, so that the boundary conditions are classified purely by the homotopy type of the data $(e, w)$ on $\partial M$. As we shall see, this framework leads naturally to a theory which describes processes involving the creation and annihilation of gravitational kinks.

At any rate, in order to complete the calculation of the sum in (8), one gauge fixes and finds that the total amplitude, $Z(M)$, is equal to the sum over all flat connections $w$ on $M$ which have the appropriate boundary conditions on $\partial M$, and that the contribution to the sum at each such flat connection $w$ is equal to the ratio of determinants

$$
\frac{(\operatorname{det} \Delta(w))^{2}}{\left|\operatorname{det} L_{-}(w)\right|}
$$

where $\Delta(w)$ is a certain Laplacian constructed using $w$, and $L_{-}(w)$ is a 'twisted' Dirac operator, also associated with $w$ (see [19]). The ratio in (9) is known as the Ray-Singer torsion, and it is a topological invariant, i.e. it does not depend on the choice of metric made in gauge fixing. Thus, the total amplitude $Z(M)$ is also manifestly a topological invariant. Therefore this toy model is a simple example of a 'topological quantum field theory'. The aim of this paper is to develop the mathematical structure so that the 'asymptotic states' in the theory can have extra, purely topological, degrees of freedom, and to interpret these degrees of freedom in terms of gravitational skyrmions. We note that this construction was first suggested in [18]. 


\section{Mathematical Framework}

As we have seen, when studying the structure of a spacetime $\left(M, g_{L}\right)$, there are two principal aspects of 'topology' which we will generically encounter: The topology of the manifold $M$ (or submanifolds of $M$ ) and the topology of the metric $g_{L}$. Two manifolds are topologically equivalent iff they are homeomorphic. Two metrics are topologically equivalent iff they are homotopic. The problem of classifying metrics up to homotopy type in $(2+1)$ dimensions has been investigated elsewhere [27]. Explicitly, in [27] the homotopy classification problem was solved on spacetimes of the form $\Sigma \times \mathbb{R}$, where $\Sigma$ is any Riemann surface. It turns out that a (non-singular) metric $g$ on $\Sigma \times \mathbb{R}$ is homotopic to another (non-singular) metric $g^{\prime}$ on $\Sigma \times \mathbb{R}$ if and only if the kink number of $g$ with respect to $\Sigma$ is equal to the kink number of $g^{\prime}$ with respect to $\Sigma$. This is to be expected, since after sll the kink number of a Lorentz metric $g$ with respect to a two-surface $\Sigma$ is precisely the degree of the map, from $\Sigma$ to $S^{2}$, defined by any timelike vector field associated with $g$. Since we are not working in the metric formulation of general relativity, but rather in the connection formalism, we must transcribe the above discussion and understand when two triads, $e$ and $e^{\prime}$, are homotopic. If $v_{0}$ denotes the vector dual to the 'timelike' 1 -form $e_{\mu}^{0}$, and $v_{0}^{\prime}$ is likewise dual to $\left(e_{\mu}^{0}\right)^{\prime}$, it is easy to see that the two triads are in the same homotopy class $\left(e \sim e^{\prime}\right)$ if and only if $\operatorname{kink}\left(\Sigma ; v_{0}\right)=\operatorname{kink}\left(\Sigma ; v_{0}^{\prime}\right)$, where again we assume that the spacetime has the form $\Sigma \times \mathbb{R}$. Henceforth, we shall simply write ' $\operatorname{kink}(\Sigma ; e)$ ' to denote the integer classifying the homotopy class of $e$ with respect to $\Sigma$ (see [25], [26] and [27] for the further properties of kinks).

What does this have to do with gravitational skyrmioms?

Well, recall that a skyrmion [29], in any field theory, is simply an extended structure corresponding to a field configuration with non-trivial topology. It therefore makes sense to use the terms 'kink' and 'skyrmion' interchangeably [25].

Suppose then, that $M$ is a three-manifold with boundary $\partial M$ equal to the disjoint union of a finite number of closed, orientable two-manifolds:

$$
\partial M \cong \Sigma_{1} \cup \Sigma_{2} \cup \Sigma_{3} \cup \ldots \cup \Sigma_{n}
$$

In general, it may be the case that there exists flat $\operatorname{ISO}(2,1)$ data $(e, w)$ on 
$M$ with non-trivial homotopy with respect to $\partial M$, i.e. for some component(s) $\Sigma_{i}$ of $\partial M$,

$$
\operatorname{kink}\left(\Sigma_{i} ; e\right) \neq 0
$$

If we take any collar neighbourhood $\Sigma_{i} \times(0,1)$ on which $e$ is non-singular, we thus see that $\operatorname{kink}\left(\Sigma_{i} ; e\right)$ classifies the homotopy type of the data on $\Sigma_{i} \times(0,1)$. If we interpret a given collar neighbourhood $\Sigma_{i} \times(0,1)$ as an 'incoming' (or outgoing) asymptotic state, we see that there are thus two pieces of topological information associated to any asymptotic state: The topology of the boundary component $\Sigma_{i}$ and the topology of the flat bundle (associated with the flat data $(e, w)$ ) relative to $\Sigma_{i}$. Let us adopt the notation

$$
\operatorname{kink}\left(\sum_{i} ; e\right)=k_{i}
$$

The we shall denote an 'incoming' state using the ket notation:

$$
\mid\left(\Sigma_{\text {in }}, k_{\text {in }}\right)>
$$

Likewise, we use bra notation for 'outgoing' states':

$$
<\left(\Sigma_{\text {out }}, k_{\text {out }}\right) \mid
$$

We wish to extend the selection rules of [21], which dealt with the special case $k_{\text {in }}=k_{\text {out }}=0$ (referred to as the 'spatial sector'). As we shall see, by allowing for skyrmion annihilation/production $\left(k_{\text {in }} \neq 0, k_{\text {out }} \neq 0\right)$, more exotic transitions are allowed.

\section{Selection Rules and Suppressed Processes}

Let $M$ be a closed three-manifold with boundary equal to the disjoint union of a finite collection of closed orientable two-manifolds $\Sigma_{i}$, as above, and let $k_{i}$ denote the kink number (with respect to a given $\Sigma_{i}$ ) of some flat $I S O(2,1)$ data $(e, w)$ on $M$. We are concerned with understanding when flat data $(e, w)$ inducing the given boundary conditions exists, since given the existence of such a connection, it necessarily follows that the amplitude (8) must be non-vanishing. Likewise, if such flat data does not exist, then the amplitude for the process must vanish. Indeed, one readily sees that the three-manifold $M$ mediating the transition is irrelevant, and that the real 
problem is thus to classify which collections of data $\left\{\left(\Sigma_{i}, k_{i}\right)\right\}$ correspond to processes with non-vanishing amplitude.

Thus, in analogy with the construction in [21], let $P_{i}$ denote an $S O(2,1)$ bundle over $\Sigma_{i}$ corresponding to the data with homotopy $k_{i}$. It follows, by standard formulae, that the Euler class of the $S O(2,1)$ bundle over $\Sigma_{i}$ depends upon both the topology of $\Sigma_{i}$ and the 'winding' of the bundle:

$$
\operatorname{eul}\left(P_{i}\right)= \pm\left(\chi_{i}-\operatorname{kink}\left(\Sigma_{i} ; e\right)\right)
$$

where $\chi_{i}=\chi\left(\Sigma_{i}\right)$.

On the other hand, the sum of all the Euler classes will still equal an integral over the boundary of a two-form corresponding to the kink density

$$
\sum_{i=1}^{n} \operatorname{eul}\left(P_{i}\right)= \pm \sum_{i=1}^{n} k_{i}
$$

where the $( \pm)$ in equation (10) is the same choice as in equation (11). We thus have proved

Theorem. Let $\left\{\left(\Sigma_{i}, k_{i}\right) \mid i=1, \ldots n\right\}$ be some collection of asymptotic states. Then

$$
<\left(\Sigma_{n}, k_{n}\right),\left(\Sigma_{n-1}, k_{n-1}\right), \ldots\left(\Sigma_{j+1}, k_{j+1}\right) \mid\left(\Sigma_{j}, k_{j}\right), \ldots\left(\Sigma_{1}, k_{1}\right)>\neq 0
$$

if and only if

$$
\sum_{i=1}^{n} \varepsilon^{i}\left(\chi_{i}-2 k_{i}\right)=0
$$

for some choice $\varepsilon^{i}= \pm 1$.

Example 1. Let $\Sigma \cong S^{2}, k=1=\operatorname{kink}(\Sigma ; e)$. Then by the above Theorem, we have

$$
<\left(S^{2}, 1\right) \mid \phi>\neq 0
$$

In other words, it is possible to nucleate a single ' $S$ ' universe' from nothing, as long as one creates a kink in the process. One can realise this process by simply embedding $S^{2}$ in $(2+1)$-Minkowski space (so that $M \cong B^{3}$ ).

Example 2. Let $\Sigma$ denote the connected sum of $n$ tori

$$
\text { tori : } \Sigma \cong \overbrace{T \# T \# \ldots \# T \text {. }}^{n \text { times }} \text {. }
$$


Let $k=-1+n$. Then again, using the Theorem, we find

$$
<(\Sigma, n-1) \mid \phi>\neq 0
$$

Again. this process can be visualised by simply embedding the handlebody $\Sigma$ in $(2+1)$-Minkowski space.

\section{Conclusion and Acknowledgements}

We have extended the work of [21] to a selection rule that governs which types of skyrmionic creation/annihilation processes are allowed in the theory. We note that such processes are only defined for Lorentzian signature; it seems that one loses information in passing to a Euclidean formalism. Presumably this issue should be explored further.

The author would like to thank Dr. G.W. Gibbons, Dr. L.J. Alty, Dr. T.J. Foxon, N. Lambert and S.F. Ross for useful discussions. Also eternal gratitude and thanks go to Jo Chamblin (Piglit) for loving support and help

with the preparation of this paper. This work was supported by NSF Graduate Fellowship No. RCD-9255644.

\section{References}

[1] Geroch R.P., Topology in General Relativity, J. Math. Phys., 8, No. 4, 1967

[2] Horowitz G.T., Topology Change in Classical and Quantum Gravity, Class. Quant. Grav., 8, 1991

[3] Chamblin A. and Penrose R., Kinking and Causality, Twistor Newsletter, 34, pgs. 13-18, 1992

[4] Bass R.W. and Witten L., Rev. Mod. Phys., 29, pg. 452, 1957

[5] Steenrod N., Topology of Fibre Bundles, Princeton Univ. Press, 1951 
[6] Hector G. and Hirsch U., Introduction to the Geometry of Foliations, Parts A and B, Aspects of Mathematics, Friedr. Vieweg \& Sohn Verlagsgesellschaft mbH, Braunsschweig, 1981

[7] Sorkin R., Consequences of Spacetime Topology, Proc. of the Third Canadian Conf. on Gen. Rel. and Rel. Astrophys.

[8] Hawking S.W., Spacetime Foam, Nucl. Phys. B, 144, pg. 349, 1978

[9] Ashtekar A., New Variables for Classical and Quantum Gravity, Phys. Rev. Lett., 57, pg. 2244, 1986

[10] Witten E., 2 + 1 Dimensional Gravity as an Exactly Soluble System, Nucl. Phys. B, 311, pg. 46, 1988

[11] Hawking S.W. amd Ellis G., The Large-Scale Structure of Spacetime, CUP, 1973

[12] Finkelstein D. and Misner C., Some New Conservation Laws, Annals of Physics, 6, pgs. 230-243, 1959

[13] Gibbons G.W. and Hawking S.W., Kinks and Topology Change, Phys. Rev. Lett., 69, No. 12, 1992

[14] Chamblin A., Gibbons G.W. and Steif, A.R., Kinks and Time Machines, Phys. Rev. D, 50, pgs. 2353-2355, 1994

[15] Borde A., Topology Change in Classical General Relativity, gr-qc preprint No. 9406053

[16] Yodzis, P., Lorentz Cobordism, Comm. Math. Phys., 26, p.39, 1994

[17] Milnor, J., Morse theory, Princeton Univ. Press, Princeton, NJ, 1951

[18] Gibbons, G.W., Topology Change: Kinks and Spinors, Proc. of the International Workshop of Theoretical Physics, 6th Session: "String Quantum Gravity and Physics at the Planck Energy Scale", Erice, Sicily, 21-28 
June (1992)

[19] Witten E., Topology Changing Amplitudes in 2+1-Dimensional Gravity, Nucl. Phys. B 323, 113-140, 1990

[20] Carlip S. and Cosgrove R., Topology Change in $(2+1)$-Dimensional Gravity, J. Math. Phys. 35, No. 10, 5477-5493, 1994

[21] Amano K. and Higuchi S., Topology Change in ISO $(2,1)$ Chern-Simons Gravity, Nucl. Phys. B 377, 218, 1992

[22] Carlip S. and de Alwis S., Wormholes in 2+1 Dimensions, Nucl. Phys. B 337, 681, 1990

[23] Ashtekar A., Husain V., Rovelli C., Samuel J., and Smolin L., $2+1$ Quantum Gravity as a Toy Model for the $3+1$ Theory, Class. Quant. Grav. 6, L185-L193, 1989

[24] Carlip S., Exact Quantum Scattering in 2+1-Dimensional Gravity, Nucl. Phys. B 324, 106, 1989

[25] Finkelstein D., Kinks, J. Math. Phys. 7, No. 7, 1218, 1966

[26] Williams J.G. and Zvengrowski P., Spin in Kink-Type Field Theories, Int. J. Theor. Phys. 16, No. 10, 755-761, 1977

[27] Williams J.G. and Zvengrowski P., Kink Metrics in (2+1)-Dimensional Spacetime, J. Math. Phys. 33, No. 1, 256, 1992

[28] Witten E., Quantum Field Theory and the Jones Polynomial, Comm. Math. Phys. 121, 351-399, 1989

[29] Skyrme T., The Origins of Skyrmions, Int. J. Mod. Phys. A 3, 27452751, 1988

[30] Alty L.J., The Generalised Gauss-Bonnet-Chern Theorem, DAMTP preprint No. R94/ 1994 
